\title{
The role of ATP in sleep regulation
}

\section{Sachiko Chikahisa * and Hiroyoshi Séi}

Department of Integrative Physiology, Institute of Health Biosciences, The University of Tokushima Graduate School, Tokushima, Japan

\section{Edited by:}

Norio K. Ishida, National Institute of Advanced Industrial Science and

Technology, Japan

\section{Reviewed by:}

Norio K. Ishida, National Institute of Advanced Industrial Science and Technology, Japan

Venkatramanujan Srinivasan, Sri

Sathya Sai Medical Educational and

Research Foundation, India

Lisa Marshall, University of Luebeck, Germany

\section{*Correspondence:}

Sachiko Chikahisa, Department of Integrative Physiology, Institute of

Health Biosciences, The University of

Tokushima Graduate School,

Tokushima 770-8503, Japan.

e-mail: chika@basic.med.tokushima-u.

ac.jp
One of the functions of sleep is to maintain energy balance in the brain. There are a variety of hypotheses related to how metabolic pathways interact with sleep/wake regulation. A major finding that demonstrates an interaction between sleep and metabolic homeostasis is the involvement of adenosine in sleep homeostasis. An accumulation of adenosine is supplied from ATP, which can act as an energy currency in the cell. Extracellularly, ATP can act as an activity-dependent signaling molecule, especially in regard to communication between neurons and glia, including astrocytes. Furthermore, the intracellular AMP/ATP ratio controls the activity of AMP-activated protein kinase, which is a potent energy regulator and is recently reported to play a role in the regulation of sleep homeostasis. Brain ATP may support multiple functions in the regulation of the sleep/wake cycle and sleep homeostasis.

Keywords: sleep, ATP, adenosine, astrocyte, AMPK

\section{INTRODUCTION: ATP AS A NEUROMODULATOR}

Sleep is thought to be essential to replenish energy sources in the brain that are depleted during wakefulness. ATP has long been recognized as an intracellular energy source in the brain and multiple other tissues, and the complex regulation of mitochondrial ATP synthesis is triggered within the human brain in response to daily activities (Kagawa, 2010). ATP has also been proposed as a putative transmitter in sensory nerves, motor nerves, and some CNS neurons (Burnstock, 2007, 2009). In the CNS, ATP signaling can regulate both excitatory and inhibitory neurotransmission. Many neurons can release ATP in an activity-dependent manner, and this molecule can act as a potent neuromodulator for neuron-neuron and neuron-glial signaling (Fields and Stevens, 2000; Fields and Burnstock, 2006; Krueger et al., 2008). ATP and its metabolites regulate multiple processes in the nervous system including sleep regulation (Burnstock et al., 2011).

Recently, it was observed in rats that ATP levels in several brain regions (frontal cortex, basal forebrain, cingulate cortex, and hippocampus) are stable during waking but exhibit a surge during the initial hours of sleep (Dworak et al., 2010). This ATP surge displays a significant positive correlation with the intensity of EEG slow wave activity (SWA; $0.5-4 \mathrm{~Hz}$ ) in non-rapid eye movement (NREM) sleep, a parameter of sleep depth and homeostatic need for sleep. These results suggest that ATP levels drastically change during sleep in several brain regions and are directly related to SWA in NREM sleep (Dworak et al., 2010). Interestingly, sleep deprivation for $3 \mathrm{~h}$ induces a significant reduction in ATP concentration in the frontal cortex and lateral hypothalamus (an area known to predominantly contain wake- and REM-active neurons) although sleep deprivation does not affect the VLPO, an area known to predominantly contain sleep-active neurons (Dworak et al., 2010). Thus, elevations in brain ATP consumption caused by prolonged wakefulness may differ depending on the brain region.

The purpose of this review is to provide an overview of the neurobiological evidence for the role of ATP in sleep regulation. In this review, we will first provide the experimental evidence for an association between adenosine (a product from AMP) and sleep regulation. A presentation of the evidence demonstrating the role of gliotransmission in sleep homeostasis as mediated by astrocytes will follow. Finally, we will address the possibility of an involvement of AMP-activated protein kinase (AMPK), a metabolic sensor, in sleep homeostasis.

\section{ADENOSINE AND SLEEP}

The hypnogenic properties of adenosine in specific brain areas have been demonstrated in many studies. Infusion of adenosine and adenosine transport inhibitors into the basal forebrain increases sleep in cats and rats (Portas et al., 1997; Methippara et al., 2005). Extracellular adenosine levels in the basal forebrain measured by microdialysis are lower during NREM and REM sleep in cats, while levels are increased during sleep deprivation and subsequently normalized during recovery sleep (Porkka-Heiskanen et al., 2000). Furthermore, SWA in NREM sleep displays a strong positive correlation with increases in adenosine levels in the basal forebrain and frontal cortex during the recovery period after sleep deprivation (Kalinchuk et al., 2011). These findings suggest that adenosine is a sleep-promoting substance, decreasing the activity of cholinergic wake-promoting neurons in the basal forebrain that are presumably responsible for the accumulation of adenosine during prolonged waking (Porkka-Heiskanen and Kalinchuk, 2011). 


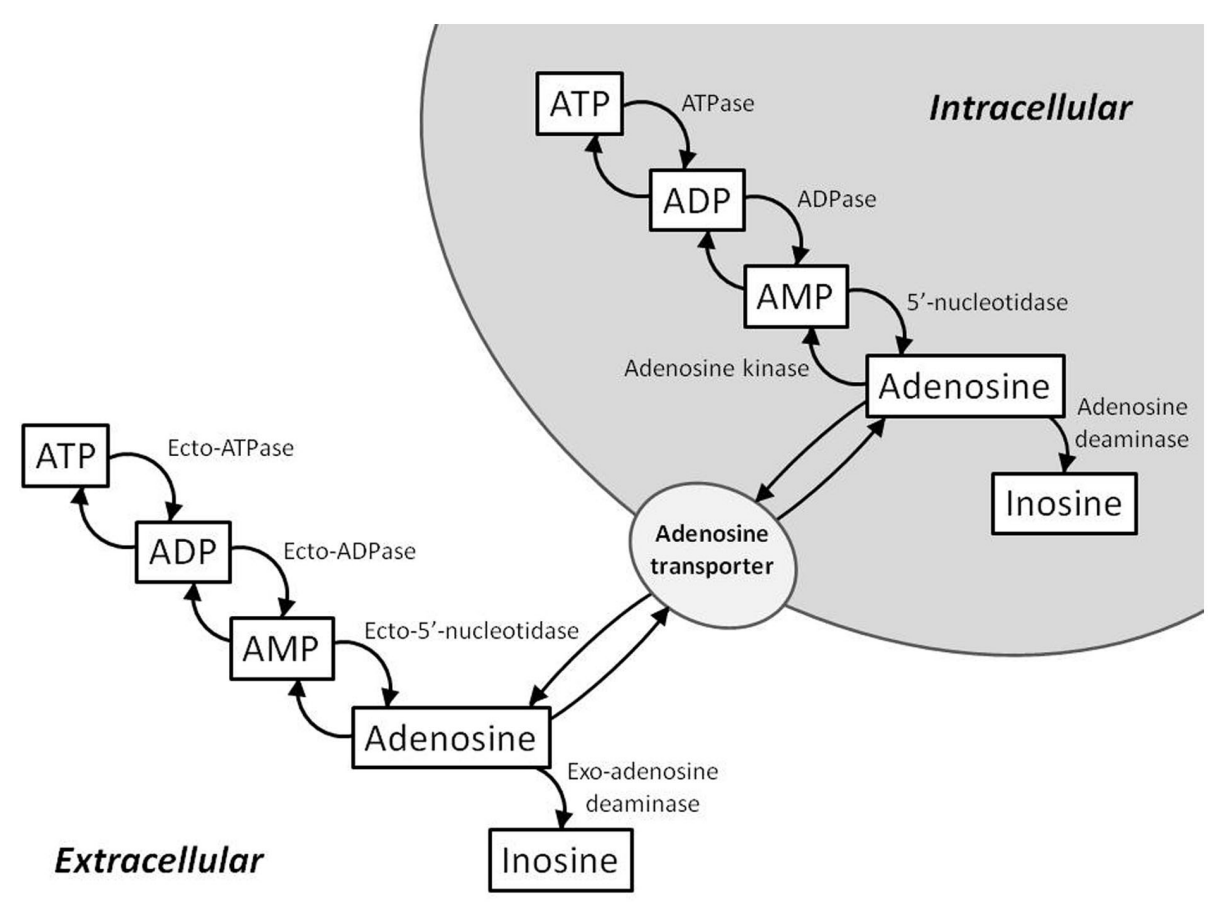

FIGURE 1 | Schematic of the intra- and extra-cellular ATP/adenosine signaling cascades. Intracellularly, adenosine is formed from AMP by cytosolic 5'-nucleotidase. Adenosine kinase and adenosine deaminase convert adenosine to AMP and inosine, respectively. Extracellularly, ATP, ADP, and AMP are converted to adenosine by ecto-5'-nucleotidases. Intracellular and extracellular adenosine levels are regulated by bi-directional equilibrative nucleoside transporters.
An increase in adenosine levels reflects an increased breakdown of cellular ATP (Figure 1). Intracellularly, ATP is metabolized to ADP and then subsequently metabolized to AMP. AMP is further metabolized to adenosine by cytosolic $5^{\prime}$-nucleotidase. Adenosine can be converted back to AMP by adenosine kinase or converted to inosine by adenosine deaminase. In terms of energy state modulation, adenosine kinase and adenosine deaminase are important regulators of intracellular and extracellular adenosine levels. Extracellularly, ATP, ADP, and AMP are converted to adenosine by ecto- $5^{\prime}$-nucleotidases. Adenosine concentration between the intra- and extra-cellular spaces is equilibrated by nucleoside transporters. When ATP is broken down in the cell, excess adenosine is transported to the extracellular space by these transporters.

The adenosinergic regulation of sleep is predominantly mediated either via $\mathrm{A} 1$ receptors (A1Rs) or A2a receptors (A2aRs). The effects of adenosine in the basal forebrain on recovery sleep mentioned above are mostly mediated by A1Rs. Perfusion of an $A 1 R$ antagonist into the basal forebrain reduces recovery sleep after sleep deprivation, while an $\mathrm{A} 2 \mathrm{aR}$ antagonist had no effect on recovery sleep in rats (Gass et al., 2009). However, evidence for a role of $\mathrm{A} 2 \mathrm{aRs}$ in the regulation of sleep has also emerged, especially concerning the actions of $\mathrm{A} 2 \mathrm{aRs}$ in the leptomeninges under the basal forebrain that are mediated by prostaglandin D2 and DP1 receptors (Urade and Hayaishi, 2011). In addition, it was reported that administration of caffeine, a non-selective adenosine receptor antagonist, induces arousal effects in wild-type and A1R-knockout mice, although its effect was not observed in A2aRknockout mice, indicating that the arousal effects of caffeine are dependent on A2aRs (Huang et al., 2005). Thus, adenosine regulates either the amount of sleep or sleep homeostasis in multiple ways depending on the brain area and receptor subtype.

\section{GLIA AND SLEEP}

Glia release multiple neuroactive substances that contribute to sleep regulation including ATP, cytokines, and glutamate (Fields and Burnstock, 2006; Krueger et al., 2008). Glia were thought to play a passive and auxiliary role in the brain. However, in the past decade, it has been reported that glia play more active roles in the brain via neuron-astrocyte interactions. Astrocytes can be activated by neurotransmitters released from active neurons they surround. In turn, astrocytes release chemical transmitters to act on neurons pre- and post-synaptically. This process of gliotransmission modulates synaptic activity, with the primary chemicals released from astrocytes being ATP, glutamate, and D-serine, all of which can act to facilitate neural transmission (Hamilton and Attwell, 2010).

Some recent studies have presented evidence that sleep homeostasis depends on gliotransmission and the accumulation of adenosine degraded from ATP. These studies use transgenic mice that express a dominant-negative ( $\mathrm{dn}$ ) SNARE domain selectively in astrocytes, and such mice have impaired gliotransmission (Halassa and Haydon, 2010; Florian et al., 2011). In the dnSNARE mice, SWA in NREM sleep at baseline and during recovery periods after sleep deprivation is attenuated compared with that observed in wild-type mice (Halassa et al., 2009). During sleep deprivation, glutamate released from presynaptic neurons binds 
to postsynaptic AMPA receptors to facilitate excitatory neurotransmission (Figure 2). Glutamate also binds to metabotropic glutamate receptors on the membranes of adjacent astrocytes and can evoke rises in intracellular $\mathrm{Ca}^{2+}$ concentration in astrocytes. In turn, astrocytes release ATP when intracellular $\mathrm{Ca}^{2+}$ is elevated. ATP released from activated astrocytes is rapidly hydrolyzed to adenosine, which reduces the release of glutamate from the presynaptic terminal by acting on A1 receptors, ultimately resulting in hyperpolarization of the postsynaptic neuron membrane potential (Jones, 2009). The dnSNARE mice, which have an impairment of gliotransmission, display an impaired regulation of sleep homeostasis, suggesting that gliotransmission is essential for sleep pressure accumulation.

It is also well known that sleep loss can induce a deficit in memory task performance in humans and rodents. The dnSNARE mice were also protected against the effects of sleep deprivation in both non-hippocampal- and hippocampal-dependent memory tasks (Halassa et al., 2009; Florian et al., 2011). In addition, i.c.v. injection of the A1R antagonist 8-cyclopentyl-1,3-dimethylzanthine (CPT) into wild-type mice mimicked the phenotype of dnSNARE mice, suggesting that sleep homeostasis and cognitive deficits are due to adenosine actions via A1Rs. Thus, astrocytes regulate sleep pressure, recovery, and associated cognitive consequences mediated by adenosine in terms of dampening neuronal excitability. Interestingly, extracellular ATP also activates purine P2 receptors (e.g. P2X7) on glial cells to release cytokines such as interleukin$1 \beta$ and tumour-necrosis factor- $\alpha$, which act as sleep regulatory substances (Krueger et al., 2008, 2010). Mice lacking functional P2X7 receptors have attenuated NREM sleep and SWA in NREM sleep during recovery periods after sleep deprivation compared with wild-type mice (Krueger et al., 2010). These data suggest that extracellular ATP and P2 receptors have an important

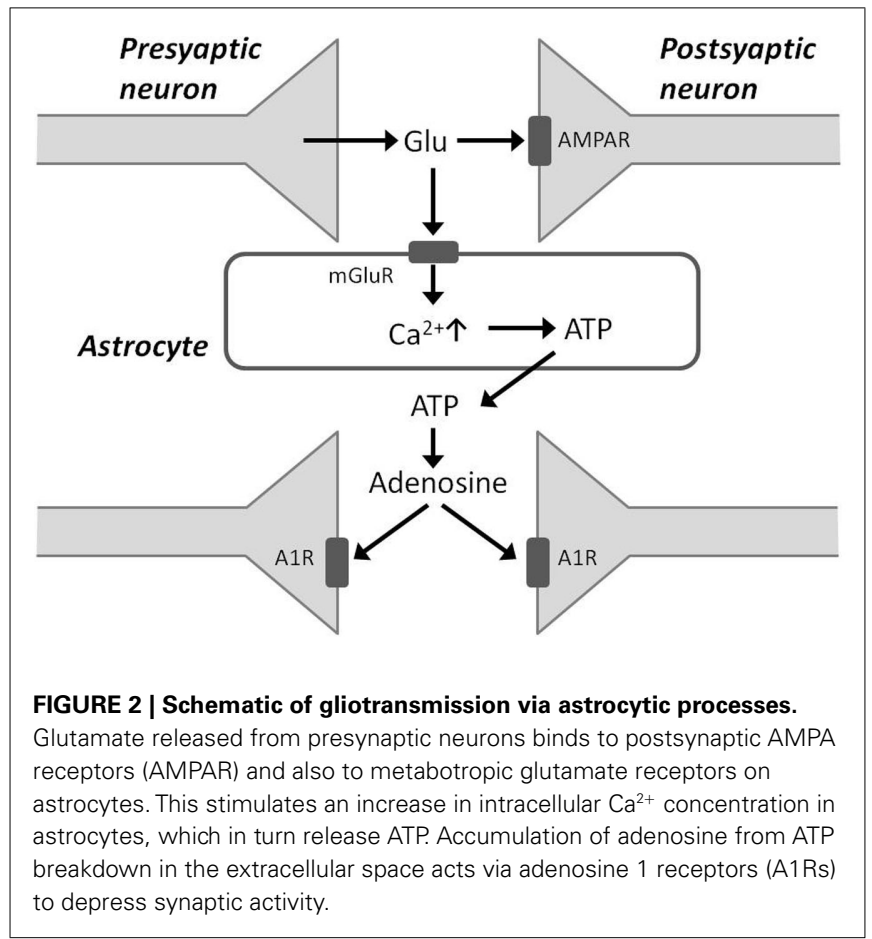

role in the regulation of sleep homeostasis and link neuro- and gliotransmission to sleep.

\section{AMPK AND SLEEP}

AMP-activated protein kinase is an efficient sensor of the cellular energy state, and regulates energy balance in the hypothalamus, liver, muscle, adipose tissue, and pancreas (Kahn et al., 2005; Minokoshi et al., 2008). AMPK is activated by phosphorylation when the intracellular AMP/ATP ratio increases, an indication that cellular energy consumed exceeds energy produced. In order to restore energy balance, activated AMPK upregulates catabolic pathways generating ATP such as fatty acid oxidation and glucose uptake, while it downregulates anabolic pathways consuming ATP such as lipid and carbohydrate synthesis. Therefore, dysfunction of peripheral AMPK leads to metabolic syndromes including type 2 diabetes and obesity (Hardie, 2008; Zhang et al., 2009).

Pharmacological experiments have indicated that energy depletion using 2,4-dinitrophenol (DNP), which blocks ATP synthesis and increases the AMP/ATP ratio, increases the amount of NREM sleep and also enhances SWA in NREM sleep (Kalinchuk et al., 2003). In agreement with this study, we have recently demonstrated that i.c.v. injection of 5-aminoimidazole-4-carboxamide riboside (AICAR), an AMP-mimicking activator of AMPK, enhances SWA in NREM sleep, while i.c.v. injection of compound C (CC), an AMPK inhibitor, suppresses SWA compared to vehicle injection in mice (Chikahisa et al., 2009). Furthermore, we have demonstrated that $6 \mathrm{~h}$ of sleep deprivation increases AMPK activity in the hypothalamus of mice (Chikahisa et al., 2009). This sleep deprivation-induced activation of AMPK is also observed in the basal forebrain, and is accompanied by decreased ATP levels in

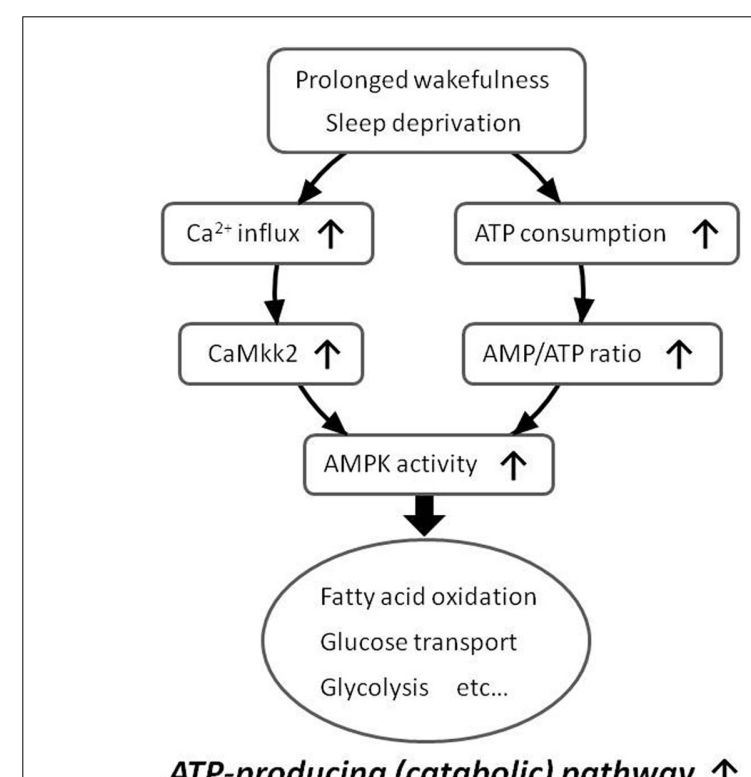

ATP-producing (catabolic) pathway $\uparrow$

FIGURE 3 | Proposed model of increased AMPK activity during prolonged wakefulness. Prolonged wakefulness such as sleep deprivation activates central AMPK via decreased AMP/ATP ratio or increased CaMkk2 expression. An activation of AMPK enhances energy-producing catabolic pathways and attenuates energy-consuming anabolic pathways. 
the same brain regions (Dworak et al., 2010). We also observed that mRNA levels of $\mathrm{Ca} 2+/$ calmodulin (CaM)-dependent protein kinase kinase $\beta$ (CaMKK2), an additional upstream kinase of AMPK, were increased immediately after $6 \mathrm{~h}$ of sleep deprivation and that carnitine palmitoyltransferase 1 (CPT1), a signaling factor downstream of AMPK, was increased during the recovery period after sleep deprivation (Chikahisa et al., 2009). These findings suggest that prolonged wakefulness enhances the activity of central AMPK by increases in the AMP/ATP ratio and/or CaMKK2 expression. Subsequent activation of central AMPK would then cause an enhancement of SWA in NREM sleep (Figure 3).

AMP-activated protein kinase is also known to be involved in circadian rhythms, and directly regulates phosphorylation and degradation of cryptochrome 1 (CRY1), a circadian clock component (Lamia et al., 2009). Furthermore, mice with a genetic disruption of the AMPK pathway show an attenuation of circadian rhythms in the expression of several clock genes in the liver (Lamia et al., 2009). Taken together, AMPK plays an important role in linking energy metabolism and the regulation of circadian and sleep homeostasis. Of course, ATP is itself affected by circadian cycles. The ATPase activity of KaiC constitutes the circadian system of cyanobacteria (Terauchi et al., 2007), while ATP release

\section{REFERENCES}

Burnstock, G. (2007). Physiology and pathophysiology of purinergic neurotransmission. Physiol. Rev. 87, 659-797.

Burnstock, G. (2009). Purinergic cotransmission. Exp. Physiol. 94, 20-24.

Burnstock, G., Krugel, U., Abbracchio, M. P., and Illes, P. (2011). Purinergic signalling: from normal behaviour to pathological brain function. Prog. Neurobiol. 95, 229-274.

Chikahisa, S., Fujiki, N., Kitaoka, K., Shimizu, N., and Sei, H. (2009). Central AMPK contributes to sleep homeostasis in mice. Neuropharmacology 57, 369-374.

Dworak, M., Mccarley, R. W., Kim, T., Kalinchuk, A. V., and Basheer, R. (2010). Sleep and brain energy levels: ATP changes during sleep. J. Neurosci. 30, 9007-9016.

Fields, R. D., and Burnstock, G. (2006). Purinergic signalling in neuron-glia interactions. Nat. Rev. Neurosci. 7, 423-436.

Fields, R. D., and Stevens, B. (2000). ATP: an extracellular signaling molecule between neurons and glia. Trends Neurosci. 23, 625-633.

Florian, C., Vecsey, C. G., Halassa, M. M., Haydon, P. G., and Abel, T. (2011). Astrocyte-derived adenosine and $\mathrm{A} 1$ receptor activity contribute to sleep loss-induced deficits in hippocampal synaptic plasticity and memory in mice. J. Neurosci. 31, 6956-6962.
Gass, N., Porkka-Heiskanen, T., and Kalinchuk, A. V. (2009). The role of the basal forebrain adenosine receptors in sleep homeostasis. Neuroreport 20, 1013-1018. Munoz, J. R., Lee, S. Y., Abel, T., Haydon, P. G., and Frank, M. G. (2009). Astrocytic modulation of sleep homeostasis and cognitive consequences of sleep loss. Neuron 61, 213-219.

Halassa, M. M., and Haydon, P. G. (2010). Integrated brain circuits: astrocytic networks modulate neuronal activity and behavior. Annu. Rev. Physiol. 72, 335-355.

Hamilton, N. B., and Attwell, D. (2010). Do astrocytes really exocytose neurotransmitters? Nat. Rev. Neurosci. 11, 227-238.

Hardie, D. G. (2008). Role of AMPactivated protein kinase in the metabolic syndrome and in heart disease. FEBS Lett. 582, 81-89.

Huang, Z. L., Qu, W. M., Eguchi, N., Chen, J. F., Schwarzschild, M. A., Fredholm, B. B., Urade, Y., and Hayaishi, O. (2005). Adenosine A2A, but not $\mathrm{A} 1$, receptors mediate the arousal effect of caffeine. Nat. Neurosci. $8,858-859$.

Jones, B. E. (2009). Glia, adenosine, and sleep. Neuron 61, 156-157.

Kagawa, Y. (2010). ATP synthase: from single molecule to human bioenergetics. Proc. Jpn. Acad. Ser. B Phys. Biol. Sci. 86, 667-693.

Kahn, B. B., Alquier, T., Carling, D., and Hardie, D. G. (2005). AMP-activated
Halassa, M. M., Florian, C., Fellin, T.,

in astrocytes is also regulated by circadian mechanisms (Marpegan et al., 2011). Circadian clock components and ATP modulation of mammalian CNS activity may cross-communicate and constitute a dual mechanism driving the regulation of AMPK as a sensor of cellular energy states.

\section{FUTURE DIRECTIONS}

Due to several advanced studies, an involvement of ATP as an energy source or as a neuromodulator in the regulation of sleep has been identified in the past few years. We now have a better understanding of the functional properties of ATP as a result of individual investigations into the properties of astrocytic, adenosine, and AMPK signaling. However, several questions still remain unanswered. SWA in NREM sleep was recently discovered to be controlled locally in the cortex (Vyazovskiy et al., 2011). Does ATP or its metabolites play a potent physiological role in sleep regulation in the cortex? Does ATP have a role in the connection between hypothalamic and cortical signaling? Further critical research to help understand the pathophysiological mechanisms of sleep disorders and the interaction between sleep impairment and metabolic syndrome is anticipated.

protein kinase: ancient energy gauge provides clues to modern understanding of metabolism. Cell Metab. 1, 15-25.

Kalinchuk, A. V., Mccarley, R. W. Porkka-Heiskanen, T., and Basheer, R. (2011). The time course of adenosine, nitric oxide (NO) and inducible NO synthase changes in the brain with sleep loss and their role in the non-rapid eye movement sleep homeostatic cascade. J. Neurochem. 116, 260-272.

Kalinchuk, A. V., Urrila, A. S., Alanko, L., Heiskanen, S., Wigren, H. K., Suomela, M., Stenberg, D., and Porkka-Heiskanen, T. (2003). Local energy depletion in the basal forebrain increases sleep. Eur. J. Neurosci. 17, 863-869.

Krueger, J. M., Rector, D. M., Roy, S., Van Dongen, H. P., Belenky, G., and Panksepp, J. (2008). Sleep as a fundamental property of neuronal assemblies. Nat. Rev. Neurosci. 9, 910-919.

Krueger, J. M., Taishi, P., De, A., Davis, C. J., Winters, B. D., Clinton, J., Szentirmai, E., and Zielinski, M. R. (2010). ATP and the purine type 2 X7 receptor affect sleep. J. Appl. Physiol. 109, 1318-1327.

Lamia, K. A., Sachdeva, U. M., Ditacchio, L., Williams, E. C., Alvarez, J. G., Egan, D. F., Vasquez, D. S., Juguilon, H., Panda, S., Shaw, R. J., Thompson, C. B., and Evans, R. M. (2009). AMPK regulates the circadian clock by cryptochrome phosphorylation and degradation. Science 326, 437-440.
Marpegan, L., Swanstrom, A. E., Chung, K., Simon, T., Haydon, P. G., Khan, S. K., Liu, A. C., Herzog, E. D., and Beaule, C. (2011). Circadian regulation of ATP release in astrocytes. $J$. Neurosci. 31, 8342-8350.

Methippara, M. M., Kumar, S., Alam, M. N., Szymusiak, R., and Mcginty, D. (2005). Effects on sleep of microdialysis of adenosine $\mathrm{A} 1$ and $\mathrm{A} 2 \mathrm{a}$ receptor analogs into the lateral preoptic area of rats. Am. J. Physiol. Regul. Integr. Comp. Physiol. 289, R1715-R1723.

Minokoshi, Y., Shiuchi, T., Lee, S., Suzuki, A., and Okamoto, S. (2008). Role of hypothalamic AMP-kinase in food intake regulation. Nutrition 24, 786-790.

Porkka-Heiskanen, T., and Kalinchuk, A. V. (2011). Adenosine, energy metabolism and sleep homeostasis. Sleep Med. Rev. 15, 123-135.

Porkka-Heiskanen, T., Strecker, R. E., and Mccarley, R. W. (2000). Brain site-specificity of extracellular adenosine concentration changes during sleep deprivation and spontaneous sleep: an in vivo microdialysis study. Neuroscience 99, 507-517.

Portas, C. M., Thakkar, M., Rainnie, D. G., Greene, R. W., and Mccarley, R. W. (1997). Role of adenosine in behavioral state modulation: a microdialysis study in the freely moving cat. Neuroscience 79, 225-235.

Terauchi, K., Kitayama, Y., Nishiwaki, T., Miwa, K., Murayama, Y., Oyama, T., 
and Kondo, T. (2007). ATPase activity of KaiC determines the basic timing for circadian clock of cyanobacteria. Proc. Natl. Acad. Sci. U.S.A. 104, 16377-16381.

Urade, Y., and Hayaishi, O. (2011). Prostaglandin $\mathrm{D}(2)$ and sleep/wake regulation. Sleep Med. Rev. 15, 411-418.

Vyazovskiy, V. V., Olcese, U., Hanlon, E. C., Nir, Y., Cirelli, C., and Tononi,
G. (2011). Local sleep in awake rats. Nature 472, 443-447.

Zhang, B. B., Zhou, G., and Li, C. (2009). AMPK: an emerging drug target for diabetes and the metabolic syndrome. Cell Metab. 9, 407-416.

Conflict of Interest Statement: The authors declare that the research was conducted in the absence of any commercial or financial relationships that could be construed as a potential conflict of interest.

Received: 29 August 2011; accepted: 07 December 2011; published online: 27 December 2011.

Citation: Chikahisa $S$ and Séi $H$ (2011) The role of ATP in sleep regulation. Front. Neur. 2:87. doi: 10.3389/fneur.2011.00087
This article was submitted to Frontiers in Sleep and Chronobiology, a specialty of Frontiers in Neurology.

Copyright (c) 2011 Chikahisa and Séi.

This is an open-access article distributed under the terms of the Creative Commons Attribution Non Commercial License, which permits non-commercial use, distribution, and reproduction in other forums, provided the original authors and source are credited. 